\title{
NEUROPSYCHOLOGICAL DEVELOPMENTS IN EPIDEMIOLOGY: TRACING THE HISTORY OF NEUROEPIDEMIOLOGY IN THE 21ST CENTURY
}

\author{
Aanya Agarwal
}

Delhi Public School, R. K. Puram

DOI: 10.46609/IJSSER.2020.v05i07.029 URL: https://doi.org/10.46609/IJSSER.2020.v05i07.029

\begin{abstract}
The allied fields of neuropsychology and neuroepidemiology have emerged as rapidly developing areas of study, research and practice. Having a shorter history than other medical disciplines, the two fields have nevertheless witnessed unprecedented technological development, growth and awareness of the diseases under their purview. There are key areas of focus which must be harnessed for a more interdisciplinary and holistic development of the two fields, to better integrate with other areas of public policy and acknowledge the inevitable sociopolitical dimensions of disease spread and treatment. Rehabilitation and improving quality of life have also emerged as key areas of focus. This paper aims to shed light on the intersection of the two fields, provide an overview of their history, and analyze current trends as well as prominent critiques of existing methods. The paper further aims to chart a course for future arenas of research, emphasizing upon the need for collaboration across disciplines and producing culturally conscious and ecologically valid studies to improve generalizability.
\end{abstract}

Keywords: Neuropsychology, Neuroepidemiology, Rehabilitation, Mental health, Disease

\section{INTRODUCTION}

Neuropsychology is an emerging discipline which aims at understanding the relationships between the brain, on the one hand, and the 'mind' and behavioral control, on the other (Berlucchi, 2009). Although humankind has always been interested in this issue, the science of neuropsychology is relatively young (Berlucchi, 2009). Its traditional approach was the study of the associations between focal brain lesions and psychological defects, but today neuropsychology is in possession of refined methodologies and theoretical frameworks for understanding both how the mind works and how the brain works (Berlucchi, 2009). Epidemiology is the study of the distribution and determinants of disease. In epidemiology, the 


\section{International Journal of Social Science and Economic Research}

ISSN: $2455-8834$

Volume: 05, Issue: 07 "July 2020"

unit of study is a person affected with a defined condition (Wallin and Kurtzke, 2014). Therefore, diagnosis is the essential prerequisite. Thus, the neurologist must be an essential part of any inquiry into Neuroepidemiology, the epidemiology of neurological diseases (Wallin and Kurtzke, 2014).

When considered together, the two emerging disciplines of neuropsychology and neuroepidemiology have increased the horizons in which diseases and particularly mental health, is studied. Tracing the historiography, progression and future development of medical disciplines is crucial as it also intersects with the social and political dimensions of disease and disease spread. Much of the information in neuroepidemiological research has been based on case series from clinic and hospital databases (Wallin and Kurtzke, 2014; Berlucchi, 2009). However, whether taken as numerator alone (case series) or compared with all admissions (relative frequency), the difficulty with such data is that one has little assurance that what has been included is representative of the total population (Wallin and Kurtzke, 2014). Such case material needs to be referenced to its proper denominator, its true source: the finite population at risk (Wallin and Kurtzke, 2014; Berlucchi, 2009).

Therefore, there are important political dimensions such as socio economic conditions of the populations at risk, the questions of who gets tested and whether parts of the population are being left out on the basis of gender, race, etc. This paper will trace the development of neuropsychology and neuroepidemiology, and provide an overview of the status of the fields. The paper will then trace the sociopolitical dimensions of the disciplines, as well as critically examine recent trends and technological developments. The paper will conclude with an overview of the potential future developments of the allied fields.

\section{BACKGROUND}

The official birth of neuropsychology as an independent scientific discipline can be dated to 1963, when an international specialty journal titled Neuropsychologia started its publication on the initiative of a small group of neurologists, psychologists, and psychiatrists partaking in an informal discussion forum called the International Neuropsychology Symposium (Berlucchi, 2009). The term 'neuropsychology' was understood to mean a particular area of neurology of common concern to neurologists, psychiatrists, psychologists, and neurophysiologists, with an interest focused mainly, but not exclusively, on the cerebral cortex, and on disorders of language, perception, and action (Berluchhi, 2009).

Cognitive neuropsychology is a subfield of cognitive neuropsychology, distinguished by the feature that it studies people with disorders of perception, attention, learning, memory, processing of spoken and written language, thinking, reasoning or belief formation, with the aim 


\section{International Journal of Social Science and Economic Research}

ISSN: $2455-8834$

Volume: 05, Issue: 07 "July 2020"

of learning more about the normal functional architectures of the cognitive processing systems used to carry out these activities (Coltheart, 2008). Hence, although it is typical for cognitive neuropsychologists to be studying people who have brain damage, these scientists are not studying the brain mechanisms associated with cognitive processes: such study is a different discipline, cognitive neuroscience (Coltheart, 2008). Cognitive neuropsychologists are studying the mind rather than the brain, the distinction of which has been a subject of debate (Coltheart, 2008).

Neuroepidemiology, a term coined nearly half a century ago, has traditionally been defined as the study of the frequency, distribution, determinants, and outcomes of neurologic diseases in human populations (Mensah et al, 2016). This definition however, highlights only the nonexperimental and descriptive aspects of neuroepidemiology (Mensah et al, 2016). As a scientific field of study, neuroepidemiology now also includes experimental aspects that span the full spectrum of clinical and population science research encompassing fundamental discovery as well as research into compelling questions and critical challenges around the translation of discovery science findings into health impact in populations with neurological disease (Mensah et al, 2016).

Traditionally, neuroepidemiology has been perceived for a long time as a science of incidence, prevalence, risk factors, natural history and prognosis of neurological disorders. However, this is only one part of neuroepidemiology, called non-experimental neuroepidemiology (Wallin and Kurtzke, 2014). The other integral, but commonly forgotten, part of neuroepidemiology is experimental neuroepidemiology, which is research based on clinical trials of effectiveness or efficacy of various interventions in neurological disorders (Wallin and Kurtzke, 2014).

Experts have noted that the dominant theories and methods used continue to be traditional despite technological advancement. A critique that is particularly pertinent to the intersection between neuropsychology and neuroepidemiology is that the assessment of disease estimates is hindered by problems in reporting for such studies (Bennett et al, 2015). Despite a growth in published reports, existing guidelines relate to analytical rather than descriptive epidemiological studies (Bennett et al, 2015). There are also no user-friendly tools (e.g., checklists) available for authors, editors and peer-reviewers to facilitate best practice in reporting of descriptive epidemiological studies for most neurological disorders (Bennett et al, 2015). This must be improved, as incidence and prevalence studies of neurological disorders play an important role in assessing the burden of disease and planning services, which intersect with other socio-political dimensions of public health policy (Bennett et al, 2015). 
International Journal of Social Science and Economic Research

ISSN: $2455-8834$

Volume: 05, Issue: 07 "July 2020"

\section{DISCUSSION}

There is promising and rapid growth in the fields of neuroepidemiology and neuropsychology, with an increasing awareness of the intersection between the two (Mensah et al, 2016; Berlucchi, 2009). With new and constantly evolving methods of neuroepidemiological studies, their wider use and increasing implementation across the globe, neuroepidemiology is expected to play a central role in the translation of evidence on the frequency, distribution and determinants of neurological disorders to clinical and public health practice in order to maximize individual patient and population health impact (Mensah, et al, 2016; Berlucchi, 2009). Over the past twenty five years, the study of these fields has also spread from North America, Europe and Australia to Asia, Africa, the Middle East, Central and South America, and New Zealand (Ponsford, 2017).

It is crucial to note that the obstacles to growth are heavily influenced by socio political dimensions and conditions (Ponsford, 2017). Obstacles have included economic constraints on health care provision, limited availability of appropriate assessment and treatment methods, linguistic diversity and illiteracy, stigma toward and/or lack of awareness of neuropsychological disorders, lack of graduate training and clinical supervision, absence of accreditation of neuropsychologists as a clinical profession, poor pay, and diminished visibility of the field within the regional culture (Ponsford, 2017). Despite these obstacles, neuropsychological research and practice is establishing itself in these regions and has grown significantly over the last quarter century (Ponsford, 2017). Major challenges remain in establishing awareness of the significance of and developing culturally appropriate methods of assessing and rehabilitating cognitive aspects of brain disorders, training programs, recognition as a profession, and dedicated funding for neuropsychology positions (Ponsford, 2017).

There have been technological developments and new trends in the fields, which have allowed for further ease and expansion of research areas and techniques. For example, publishing companies have moved to digital recording and scoring of some select neuropsychological tests (Brown and Bigler, 2018). This has added the capability to integrate response time information and with accuracy in more elegant ways than in the past (Brown and Bigler, 2018). It has also improved the scoring of complex configural results from clinical datasets. Other major developments have included a computerized cognitive battery that is based on iPad technology, teleneuropsychological assessment, where patients are assessed remotely through telephone and video, cell phone recording apps, where researchers can obtain data in the real world in real time from patients, virtual reality systems, and wearable monitors and multi-sensory devices that allow us to record multimodal data in real time outside of the clinic (Brown and Bigler, 2018). 


\section{International Journal of Social Science and Economic Research}

ISSN: $2455-8834$

Volume: 05, Issue: 07 "July 2020"

Neuroimaging has taken front and center at looking at how to image the brain and not only image pathology, but image normal development and allow one to analyze the brain with advanced imaging tools and technologies (Brown and Bigler, 2018). It has been noted that functional imaging technology has had a great effect on moving forward affective and social neuroscience, because it has provided another way to ask questions about feelings, emotions and other states that were previously ignored in the field (Brown and Bigler, 2018). Experts in the field have noted a shift towards the development of neuropsychological batteries that can be used both within an imaging context, so that data can be obtained from functional brain imaging, and can be used outside of the imaging context in the clinic (Brown and Bigler, 2018). This will allow a greater translation and integration of information from brain imaging to neuropsychological assessment (Brown and Bigler, 2018).

In order to further deepen the study of neuropsychology and neuroepidemiology in a more sociologically oriented manner, a more interdisciplinary approach is crucial to adequately capture the socio political dimensions of disease spread, which is heavily dependent on government policies, urban and rural planning, as well as discrimination, gender equality, and other such considerations (Wallin and Kurtzke, 2014). This would also assist in improving generalizability of results, as well as improve rehabilitation and treatment efforts that must necessarily stem from the knowledge gained (Wallin and Kurtzke, 2014; Bennett et al, 2015).

Effective development of rehabilitation treatments is the need of the hour, and the direction for the future. Outcome measures for clinical trials in neurology could be addressed by recording the effect of illness on the patient's daily life. In this way, the change in quality of life caused by treatment can be recorded (Savettieri, 2008). There is also a need for collaboration between specialists in neuroepidemiology and neuropsychology, with clinicians who work in neurology and with general health professionals who refer to epidemiology and to evidence-based medicine (Savettieri, 2008, Hartlage and Long, 2009). With improved awareness and education, effectiveness of communication will be enhanced across these specialties (Hartlage and Long, 2009).

\section{CONCLUSION}

Given the rapid growth and technological impact, there is great potential for the fields of neuropsychology and neuroepidemiology to develop in the future, in a more culturally inclusive and sociologically conscious manner. Turning descriptive, analytical and experimental neuroepidemiological data into clinical and public health action for population health impact requires quality data, rigorous evidence synthesis, and the design and execution of rigorous dissemination and implementation research that incorporate the dynamics and complexities of the health care system context in which interventions are delivered (Mensah et al, 2016). 


\section{International Journal of Social Science and Economic Research}

ISSN: $2455-8834$

Volume: 05, Issue: 07 "July 2020"

While the evidence base is already robust with considerable clinical translation around some of these diseases such as stroke, other illnesses like multiple sclerosis, Parkinson's, dementia and systemic diseases with neuroimmune manifestations such as systemic lupus erythematosus would be the key arenas for future research and study (Neuroepidemiology, n.d). The focus must also move in the direction of development of neuropsychological assessment in countries where these methods are now just emerging (Neuroepidemiology, n.d). Sociological approaches must entail that neuropsychology and epidemiology move to ecologically valid tests that are culturally relevant to countries that are just now beginning to develop neuropsychological communities (Brown and Bigler, 2018).

Studies of cognitive alterations from brain damage will perhaps continue to occupy a central place on the scene, but progress will inevitably depend on many other experimental and theoretical approaches and on combined contributions from all biological sciences, from molecular biology to paleoanthropology, as well as from psychology, from cognitive and computer science, and from philosophy (Berlucchi, 2009). As stated above, improving rehabilitative services in a sociologically and culturally conscious manner is key.

The fields of neuropsychology and epidemiology must move away from drug intervention trials and industry-sponsored research into drug effectiveness (Neuroepidemiology, n.d). Independently funded cohort studies reporting real-world surveillance of pharmaceutical agents in neurological disease must be encouraged, provided other relevant risk factors associated with health outcomes, particularly lifestyle risk factors, are reported and controlled for (Neuroepidemiology, n.d). Neuroepidemiology must be committed to developing and strengthening the evidence base around modifiable factors that can be addressed in a preventive medicine model to improve the health outcomes of people with a range of neurological conditions that are currently not considered curable (Neuroepidemiology, n.d).

There is an urgent need for research that can lead to evidence-based recommendations for practice. Important among these are comparative effectiveness studies and large, simple, pragmatic trials that will generate practice-based evidence (Mensah et al, 2016; Ponsford, 2017; Savettieri, 2008). Concerted, coherent, and timely translation of the knowledge generated into evidence-based recommendations for practice with corresponding changes in health policy at the governmental levels will be crucial (Mensah et al, 2016). So will early and sustained engagement of multiple, diverse stakeholders including public and private sponsors of research together with academic, community, delivery system, and public health representatives and decision-makers in order to reduce the burden of such diseases worldwide (Mensah et al, 2016). 
International Journal of Social Science and Economic Research

ISSN: $2455-8834$

Volume: 05, Issue: 07 "July 2020"

\section{REFERENCES}

APA Journals Dialogue Episode 27', American Psychological Association, https://www.apa.org/pubs/highlights/podcasts/episode-27

Bennett, DA, et al, (2015), 'Development of the Standards of Reporting of Neurological Disorders (STROND) Checklist: A Guideline for the Reporting of Incidence and Prevalence Studies in Neuroepidemiology', European Journal of Epidemiology, Vol.30, Issue 7, pp.569-76

Berlucchi, G, (2009), 'Neuropsychology: Theoretical Basis', Enyclopaedia of Neuroscience, https://www.sciencedirect.com/science/article/pii/B9780080450469009967

Brown, G, and Bigler, E, (2018), 'Neuropsychology: Introduction to a Special Issue

Coltheart, M, (2008), ‘Cognitive Neuropsychology', Scholarpedia, Vol.3, Issue, 2, p.3644

Hartlage, L, and Long, C, (2009), 'Development of Neuropsychology as a Professional Psychological Specialty: History, Training, and Credentialing', in C.R. Reynolds, E. Fletcher-Janzen (eds.), Handbook of Clinical Child Neuropsychology, DOI 10.1007/978$0-387-78867-8 \_1$

Mensah, G, et al, (2016), 'From Data to Action: Neuroepidemiology Informs Implementation Research for Global Stroke Prevention and Treatment', Neuroepidemiology, Vol.45, Issue 3, pp.221-229

Neuroepidemiology, (n.d), 'Frontiers in Neurology: Scope', https://www.frontiersin.org/journals/neurology/sections/neuroepidemiology\#about

Ponsford J. International growth of neuropsychology. Neuropsychology. 2017;31(8):921-933. doi: $10.1037 /$ neu0000415

Savettieri, G, (2008), 'Neuroepidemiology not only for neuroepidemiologists', The Lancet, Vol. 7 Issue 5, p.388, https://www.thelancet.com/journals/laneur/article/PIIS1474$\underline{4422(08) 70083-8 / \text { fulltext }}$

Wallin, MT, and Kurtzke, JF, (2014), 'Neuroepidemiology: Overview', Encyclopaedia of the Neurological Sciences (Second ed.), pp. 383-388 